\title{
Die Politisierung der Stadtplanung: die performative Rolle von Planungsinstrumenten in Konfliktzonen am Beispiel Jerusalem
}

\author{
Peter Van Gielle Ruppe $\cdot$ Ilse Helbrecht $\cdot$ Peter Dirksmeier
}

Eingegangen: 19. Dezember 2011 / Angenommen: 6. August 2012 / Online publiziert: 7. September 2012

(C) Die Autor(en) 2012. Dieser Artikel ist auf Springerlink.com mit Open Access verfügbar.

Zusammenfassung Die politische Instrumentalisierung der Stadtplanung ist ein in der gegenwärtigen Planungswissenschaft nur wenig beachtetes Feld. Die Vorstellung einer Stadtplanung als unvoreingenommenes und rationales Instrument der Verwaltung zum räumlichen Ausgleich und zur Verbesserung von Lebensbedingungen wird in jüngster Zeit zunehmend durch kritische Positionen in Bezug auf die gesellschaftliche Rolle der Stadtplanung, ihre Kontextgebundenheit, ihre Legitimation, ihren Auftrag und damit auch ihren Bezügen zu privaten Interessen und politischer ebenso wie ökonomischer Macht ergänzt. Jerusalem als Hauptstadt Israels kann als ein prototypisches Beispiel für die politische Instrumentalisierung der Stadtplanung dienen. Der Beitrag nimmt eine skalare Betrachtung der politischen Folgen stadtplanerischen Handelns in Jerusalem vor und analysiert die wechselseitigen Relationen zwischen lokalen, nationalen und geostrategischen Interessen und Interventionen, die in den Praxen der relevanten Akteure ihren Ausdruck finden. Der Aufsatz kommt auf der Grundlage postkolonialer Geographien und performanztheoretischer Ansätze zu dem Ergebnis, dass die Jerusalemer Stadtplanung als politisches Instrument einen bedeutenden Anteil an der zu beobachtenden performativen Implementierung geographischer Imaginationen von Jerusalem als vereinigte Hauptstadt des Staates Israel und Symbol der jüdischen Nation aufweist. Damit fungiert die

Prof. Dr. I. Helbrecht $(\bowtie) \cdot$ P. Van Gielle Ruppe ·

Dr. P. Dirksmeier

Geographisches Institut, Humboldt-Universität zu Berlin,

Unter den Linden 6, 10099 Berlin, Deutschland

E-Mail: ilse.helbrecht@geo.hu-berlin.de

P. Van Gielle Ruppe

E-Mail: Peter.van.Gielle.Ruppe@geo.hu-berlin.de

Dr. P. Dirksmeier

E-Mail: peter.dirksmeier@geo.hu-berlin.de
Jerusalemer Stadtplanung als ein aktiver Agent der Durchsetzung hegemonialer politischer Interessen, die weit über den lokalräumlichen Kontext (Ost)Jerusalems hinausgehen.

Schlüsselwörter Politische Stadtplanung · Jerusalem · Geographische Imaginationen · Performanztheorie · Nahostkonflikt · Skalare Geographie

\section{Marking Politics Through Urban Planning: On the Performative Role of Planning Tools in Contested Terrain-The Case of Jerusalem}

Abstract The utilization of urban planning practices for political purposes has become an issue of planning theory in the last decades. Until the 1970s town planning was considered to be a neutral and rational instrument for the purpose of spatial regulation and administration as well as the general improvement of living conditions. Only recently with the rise of communicative planning theory this perspective has been complemented by critical perceptions of the political and ideological role of planning practices within society, their context-dependence, legitimization, normative purposes and also its interlinkages to individual aims as well as political and economic power. Jerusalem, the (partly occupied) capital of Israel, is a prototypic example of the utilization of urban planning for (even geo) political ends. This article is based on qualitative fieldwork and aims to scrutinize the scalar political consequences of various urban planning practices in Jerusalem. We undertake an analysis of the reciprocal relations of local, national, and geostrategic interests as well as the resulting actions by the relevant stakeholders. Theoretically informed by postcolonial geographies and performative approaches the paper concludes, that urban planning in Jerusalem is a highly political instrument that 
is strongly involved in the performative implementations of particular geographic imaginations of Jerusalem as a united capital of the state of Israel and historic as well as religious symbol of the Jewish nation. Hence urban planning in Jerusalem is an active element in the enforcement of hegemonic political interests which reach beyond the local context of Eastern Jerusalem.

Keywords Urban planning · Politics · Jerusalem · Geographic imaginations · Performance ·

Arab-Israeli conflict $\cdot$ Politics of scale

\section{Einleitung}

Während Stadtpolitik traditionell mit Fragen der Macht, Verteilung und Interessengeleitetheit verbunden ist (vgl. Häußermann/Läpple/Siebel 2008: 339), galt Stadtplanung bis in die frühen 70er Jahre hinein als vorwiegend rationale und ingenieurstechnische Intervention im städtischen Raum. Gerade in demokratisch organisierten Gesellschaften zielte das Handeln der lokalen Administration in Ausübung des parlamentarischen Willens darauf - so das Selbstbild traditioneller Planung -, dem Allgemeinwohl zu dienen. Die Orientierung am Gemeinwesen und damit an einem vermeintlich rationalen, übergeordneten Nutzen planerischer Maßnahmen waren wesentliches Motiv und Zielgröße planerischen Handelns. Dieses ursprünglich szientifisch-zweckrationale Planungsmodell hat Diskussionen zu Machtfragen und der Instrumentalisierbarkeit der Planung für andere Zwecke lange Zeit von sich aus quasi ausgeschlossen (vgl. Reuter 2004). Vielmehr galt der Planer als Ingenieur und Techniker, der sich auf wissenschaftliche Analysen stützt und dem rationaleren Argument folgt oder dieses präsentiert. Die Polarisierung der Aufgabenteilung zwischen einer rationalen, neutralen Planung auf der einen Seite, in der Fachleute sachliche Belange abwägen und entscheiden, und einer normativen Stadtpolitik auf der anderen Seite, die im Parlament Interessenkonflikte diskutiert und in der Regierungsarbeit auf Basis von Mehrheiten entscheidet, hat das Selbstverständnis vieler Generationen von Planern in den westlichen Ländern geprägt (vgl. Albers/Papageorgiou-Venetas 1984).

Erst langsam setzte seit den 70er Jahren nach dem Ende der Planungseuphorie eine Debatte ein um die gesellschaftliche Rolle der Stadtplanung, ihre Kontextgebundenheit, ihre Legitimation, ihren Auftrag und damit auch ihre Bezüge zu privaten Interessen und politischer ebenso wie ökonomischer Macht. Insbesondere seit den frühen 90er Jahren hat sich durch die Erweiterung der Planungsinstrumente und die Wende hin zu einer kommunikativen, auf Moderation basierenden Planung das Selbstbild der Planer grundlegend gewandelt (Helbrecht 1994; Selle 1994). So führt Deike Peters in ihrer Analyse des aktuellen Stands der Planungstheorie aus: „Die wichtigste Wandlung der planungstheoretischen Zunft seit ihren Anfängen in der Mitte des 20. Jahrhunderts bis ins aktuelle frühe 21. Jahrhundert ist sicherlich die Reifung von einer vornehmlich technokratisch verstandenen Wissenschaft ... hin zu einem mehrheitlich interpretativ-kommunikativ verstandenen Forschungszweig" (Peters 2004: 5). Uwe Altrock, Simon Güntner und Corinna Kennel sprechen von einer „,nachrationalistischen Planungswelt" (Altrock/Güntner/Kennel 2004: 189). Diese sei gekennzeichnet durch die Anerkennung der Verflochtenheit privater und öffentlicher Akteure ebenso wie Interessen, weshalb die neuen kooperativen Steuerungsformen zumeist unter dem Begriff der Governance oder der „Urban-Regime“-Theorie konzeptionalisiert werden (vgl. Fürst 2007; Häußermann/Läpple/Siebel 2008; Helbrecht 2009; Streich 2011).

In diesem Beitrag untersuchen wir die politische Instrumentalisierbarkeit der Stadtplanung für politische, zu Teilen nationale und geopolitische Zwecke. Ziel unseres Beitrages ist es, die tatsächlichen Wirkungsweisen und möglichen Performativa der Planung als politischem Machtinstrument vertieft zu untersuchen. Denn während es inzwischen ausgereifte planungstheoretische Reflektionen zu den Herausforderungen einer Stadtplanung als Moderation im Spielfeld unterschiedlicher privater oder ökonomischer Interessen gibt, wird in der Planerzunft noch kaum über eine direkte Instrumentalisierung von Stadtplanung für Zwecke staatlicher Machtausübung diskutiert. Zwar bestehen zahlreiche Arbeiten, die sich mit einzelnen Aspekten der Wechselwirkungen zwischen Stadtplanung und Politik befassen. Diese betreffen bspw. die Rolle der Politikberatung in der Stadtentwicklung (Pahl-Weber 2011; Sinz 2011), den ungleichen ökonomischen Nutzen von Stadtplanung für verschiedene Teile der Bevölkerung (Emmi/Santigosa 1989), ihren Missbrauch zwecks der Legitimierung von sozialer Kontrolle (Aguilar 1987) oder die kleinräumliche Umsetzung diskriminierender Ideologien wie der Rassentrennung (Christopher 1987). Eine Gesamtbetrachtung von Stadtplanung als Fortsetzung der „Politik mit anderen Mitteln“ bleibt jedoch aus. Die spezifische, umfassende Verbindung von Planung und offizieller, staatlicher Politik ist weitgehend aus dem Blickfeld wissenschaftlicher Diskussionen gerückt. Dies geschieht unseres Erachtens zu Unrecht. Denn sowohl eine szientifische wie auch eine post-szientifische Planungspolitik bleiben als staatliches, bürokratisches Handeln stets direkt eingebunden in politische Prozesse der Repräsentation, Verteilung, Dominanz und Machtausübung - bis hin zur Okkupation.

Wir wählen deshalb in diesem Beitrag ein sprechendes Beispiel, das in der Planungsliteratur schon des Öfteren als Fallstudie für mögliche Wechselverhältnisse von Stadtplanung und Politik verwendet wurde: Jerusalem (vgl. Brik 
1996; Altrock 1998; Rubin 2011). Mit einem neuen theoretisch informierten Blick, unter anderem angeregt durch Denkweisen postkolonialer Geographien und Ansätze der Performanztheorie, fragen wir nach den Wirkungsweisen der Stadtplanung in Jerusalem als politischem Instrument der Macht. Der in performanztheoretischen Ansätzen und in unserer Analyse der Jerusalemer Stadtplanung zentrale Begriff des Performativen (vgl. z. B. Schechner 1998; Schumacher 2002; Fischer-Lichte 2004; Butler 2006) bringt die Tatsache zum Ausdruck, dass Zeichen, Zeichengebrauch und die Effekte menschlicher körperlicher Präsenz über ihre beschreibende Dimension hinaus faktische Effekte aufweisen, die manifeste Veränderungen bewirken (Winkler 2004: 39). Wir verwenden darüber hinaus den Begriff ,politische Stadtplanung“, um anhand der Jerusalemer Situation eine Systematisierung der Formen der Inanspruchnahme planerischer Instrumente für politische Zwecke aufzuzeigen. Hierbei geht es uns zentral um eine skalare Betrachtung der politischen Folgen stadtplanerischen Handelns. Die wechselseitigen Relationen zwischen lokalen, nationalen und geostrategischen Maßstabsebenen, Interessen und Interventionen werden in diesem Beitrag anhand der Hauptstadt Israels als Fallstudie analysiert. Diese starke Verflechtung von Stadtplanung und Politik und die damit ausgeprägte (geo)politische Bedeutung von Stadtplanung lassen sich am Beispiel Jerusalem besonders augenfällig argumentieren. Aufgrund der besonderen historischen Umstände und kulturellen Bedeutung der Stadt sowie sich daraus ergebender Polarisation stellt Jerusalem innerhalb des westlich-demokratisch geprägten Diskurses im Hinblick auf Stadtplanung zweifellos einen Sonderfall dar. Gleichzeitig entfaltet erst dieses Szenario, trotz einer konstitutionell erklärten Allgemeindienlichkeit der Politik, das Potenzial zu einer asymmetrisch wirkenden Instrumentalisierung von Stadtplanung.

Insbesondere durch die Anwendung theoretischer Ansätze aus der neuen Kulturgeographie kann eine Analyse der durchdringenden (geo)politischen Wirkungsweisen der Stadtplanung auf unterschiedlichen räumlichen Maßstabsebenen gelingen. Hierzu greifen wir konzeptionell unter anderem auf die von Derek Gregory (1994) in Anlehnung an David Harvey entwickelte Konzeption der geographischen Imagination zurück. Untersucht man gesellschaftliche Effekte der Stadtplanung mit kulturgeographischen Ansätzen, so wird sichtbar, welch auffälliges gesellschaftliches Machtpotenzial stadtplanerische Handlungsweisen bergen. Am Ende dieses Aufsatzes soll deutlich geworden sein, dass Stadtplanung bei entsprechenden Rahmenbedingungen und dem vorhandenen Willen zu ihrer diesbezüglichen Vereinnahmung enormes politisches Potenzial aufweist. Dabei basiert - wie in diesem Beitrag gezeigt wird - die Wirkungsmacht der Stadtplanung als politischem Instrument v. a. auf der gesellschaftlichen
Bedeutung von Raumproduktionen und geographischen Imaginationen. Mittels stadtplanerischer Maßnahmen wie Straßenbahn- und Wohnungsbau, Infrastrukturprojekte oder Stadtraum-Möblierung werden ganz spezifische, z. T. religiös motivierte Raumvorstellungen in das physische Stadtbild Jerusalems eingeschrieben. Dieser Einschreibungsprozess verändert nicht nur die Physis der Stadt, er schafft in einem iterativen, performativen Prozess ein Jerusalem als stadtplanerisches Produkt einer spezifischen Raumimagination.

Zur Darstellung dieses Sachverhaltes wird das Fallbeispiel israelischer Stadtplanung in Ostjerusalem herangezogen. In diesem politisch nach wie vor umstrittenen Territorium findet eine Instrumentalisierung stadtplanerischer Maßnahmen zur Konsolidierung der israelischen Kontrolle über das Stadtgebiet statt. Über dieses Ziel sowie die damit einhergehenden Bestrebungen der Gestaltung einer israelischen Hauptstadt mit entsprechend dominierendem jüdischem Charakter ist in der Literatur weitgehend Einigkeit vorzufinden (u. a. Dumper 1996; Khamaisi 1997; Klein 1999; Bollens 2000). Weitere Standardwerke, die sich mit dieser Thematik ausführlich auseinandersetzen, sind Weizman (2007) und Gordon (2008).

In diesem Aufsatz greifen wir auf diese Literatur zurück und gehen über diese hinaus auf der Basis von Dokumentenrecherchen und acht qualitativen Experteninterviews im Herbst 2010. Die Expertengespräche wurden mit israelischen und palästinensischen Repräsentanten aus den Bereichen Politik, Medien und Forschung geführt, um ein möglichst umfassendes Bild der Situation einzufangen. Da Vertreter der israelischen Seite sich oftmals dem Interview verweigerten und stattdessen auf offizielle Regierungsdokumente verwiesen, wurden fünf von acht Interviews mit Palästinensern geführt. Um mehr Informationen über die israelischen Motive, Taktiken und Strategien zu erhalten, wurden offizielle Regierungsdokumente ausgewertet. ${ }^{1}$

Im Folgenden werden die Grundzüge einer „politischen Stadtplanung" am Beispiel Ostjerusalems dargestellt (Kap. 2). Wir verwenden den Terminus ,politische Stadtplanung" bewusst, um sprachlich explizit auf die normativen Bezüge planerischen Handelns zu verweisen. Abschließend (Kap. 3) findet eine kulturgeographische Deutung des empirischen Materials mit Hilfe postkolonialer und performanztheoretischer Ansätze statt. Hier wird die Wirkungsweise politischer Stadtplanung in Jerusalem als performativer politischer Prozess in Jerusalem interpretiert.

\footnotetext{
${ }^{1}$ Bei der Referenzierung der Interviews werden das Kürzel Int. sowie, zwecks Anonymisierung, die Initialen der entsprechenden Gesprächspartner angeführt.
} 
Abb. 1 Territoriale Entwicklung Israels. (Quelle: Israel Ministry of Foreign Affairs (2011), Bearbeitung: Peter Van Gielle Ruppe)

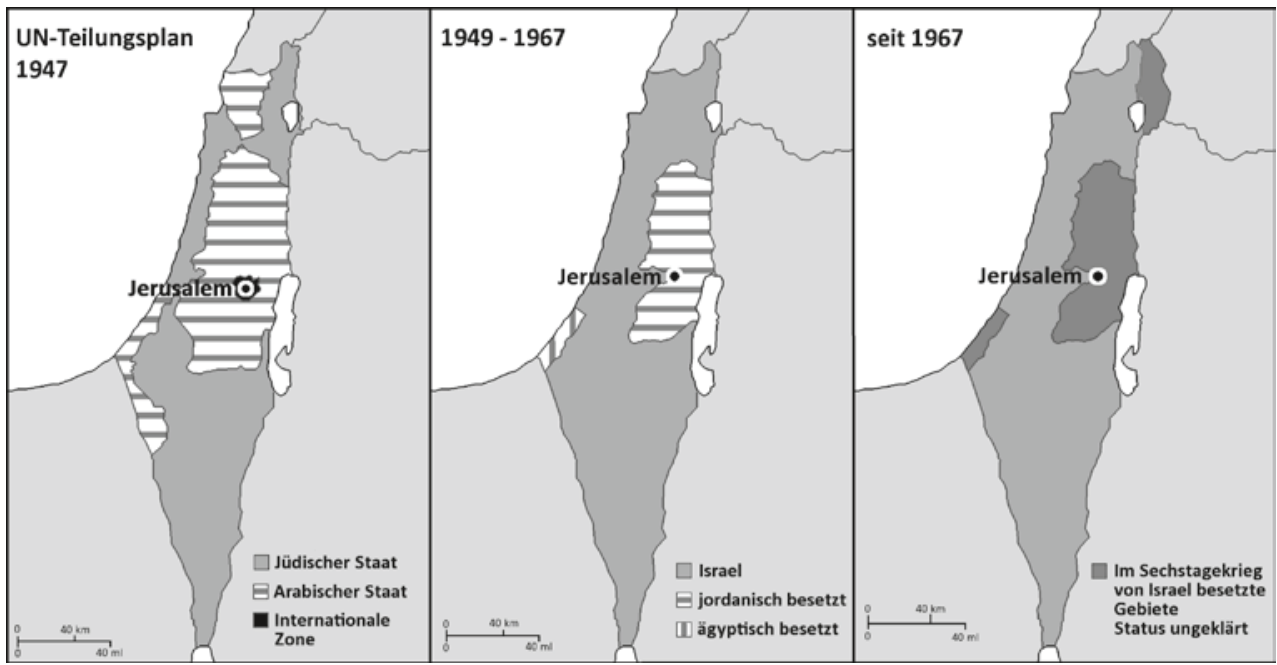

\section{Politische Stadtplanung in Jerusalem}

\subsection{Historischer Kontext}

Im Jahr 1947 legten die Vereinten Nationen (United Nations - UN) ihren Teilungsplan für das Mandatsgebiet Palästina vor, der das Territorium zwischen der zugewanderten jüdischen und der autochthonen palästinensischen Bevölkerung aufteilte. Aufgrund der besonderen Bedeutung Jerusalems für beide Parteien, beschloss die Resolution 181 (1947: Part III) der UN-Generalversammlung einen Kompromiss. Die Stadt sollte demnach den Sonderstatus eines Corpus separatum unter internationaler Verwaltung innehaben (vgl. Abb. 1), der wiederum von einem lokalen Gesetzgeberrat, zusammengestellt durch Verhältniswahlen der Stadtbewohner, regiert werden würde. Ziel war es, den Zugang zur Stadt mit ihren heiligen Stätten sowie ihre Nutzung als Handels- und Lebensraum für die Bürger beider Staaten frei und gleichberechtigt zu regeln.

Obwohl die Vertreter des zukünftigen Staates Israel den Vorschlag akzeptierten, wurde er nicht in die Tat umgesetzt, da sich das „Hohe Arabische Komitee“ - als vorstaatliche Vertretung der Palästinenser - dagegen aussprach. Massiven Rückhalt hierfür fand es bei den Regierungen der umliegenden, arabisch geprägten Staaten, die unmittelbar nach Ausruf der Unabhängigkeit Israels am 14. Mai 1948 mit Kriegserklärungen reagierten. Der Palästinakrieg endete im Juli 1949 mit einem militärischen Sieg Israels und brachte im Vergleich zum ursprünglichen UN-Teilungsplan enorme territoriale Gewinne. Israel erhielt unter anderem den westlichen Teil des Jerusalemer Stadtgebiets, während der östliche Teil Jerusalems sowie das heutige Westjordanland unter die Kontrolle Jordaniens gelangten. Abbildung 1 verdeutlicht diese territoriale Entwicklung. Trotz erneut vorausgehender Versuche der UN, den Teilungsplan umzu- setzen, wurde 1952 die Anwendung israelischen Rechts in Westjerusalem von der internationalen Staatengemeinschaft akzeptiert (Israel Ministry of Foreign Affairs 1999).

Für eine Dauer von 19 Jahren war Jerusalem durch einen sogenannten Todesstreifen geteilt. Der militärische Erfolg Israels im Sechstagekrieg 1967 erlaubte die Besetzung Ostjerusalems sowie des Westjordanlands. Die Zusammensetzung der Bevölkerung in beiden Stadthälften Jerusalems war zu dieser Zeit jeweils ethnisch homogen. Während Westjerusalem größtenteils von jüdischen Israelis bewohnt wurde, bestand die Population Ostjerusalems fast ausschließlich aus Palästinensern.

Unmittelbar nach Kriegsende begann der Staat Israel, auf die Struktur Ostjerusalems baulich Einfluss zu nehmen, zunächst in der historischen Altstadt, dann mit dem Bau der ersten Siedlungen einige Jahre darauf im gesamten Stadtgebiet (vgl. Abb. 2). Im Jahr 1980 verabschiedete die Knesset das Jerusalemgesetz und annektierte damit den östlichen Teil der Stadt nach israelischer Rechtslage (Israel Ministry of Foreign Affairs 1980). Die internationale Anerkennung der Annexion blieb aus. Während der westliche Teil der Stadt heute also international anerkannt dem Staat Israel zugehört, ist der östliche Teil Jerusalems nach internationaler Rechtslage weiterhin lediglich von Israel besetzt. Dennoch untersteht das gesamte Stadtgebiet faktisch der Kontrolle Israels. Das großteils von Palästinensern bewohnte Ostjerusalem fällt damit unter die Zuständigkeit der israelischen Stadtverwaltung. Einer endgültigen Klärung des Status Jerusalems stehen divergierende politische Interessen entgegen, da die palästinensische Bevölkerung Israel nicht als Souverän anerkennt und die Palästinensischen Autonomiebehörden den östlichen Teil der Stadt, gemäß des ursprünglichen Grenzverlaufs zwischen Israel und Jordanien vor dem Sechstagekrieg, als Hauptstadt eines zukünftigen Palästinenserstaates beanspruchen. Die Problematik bei 


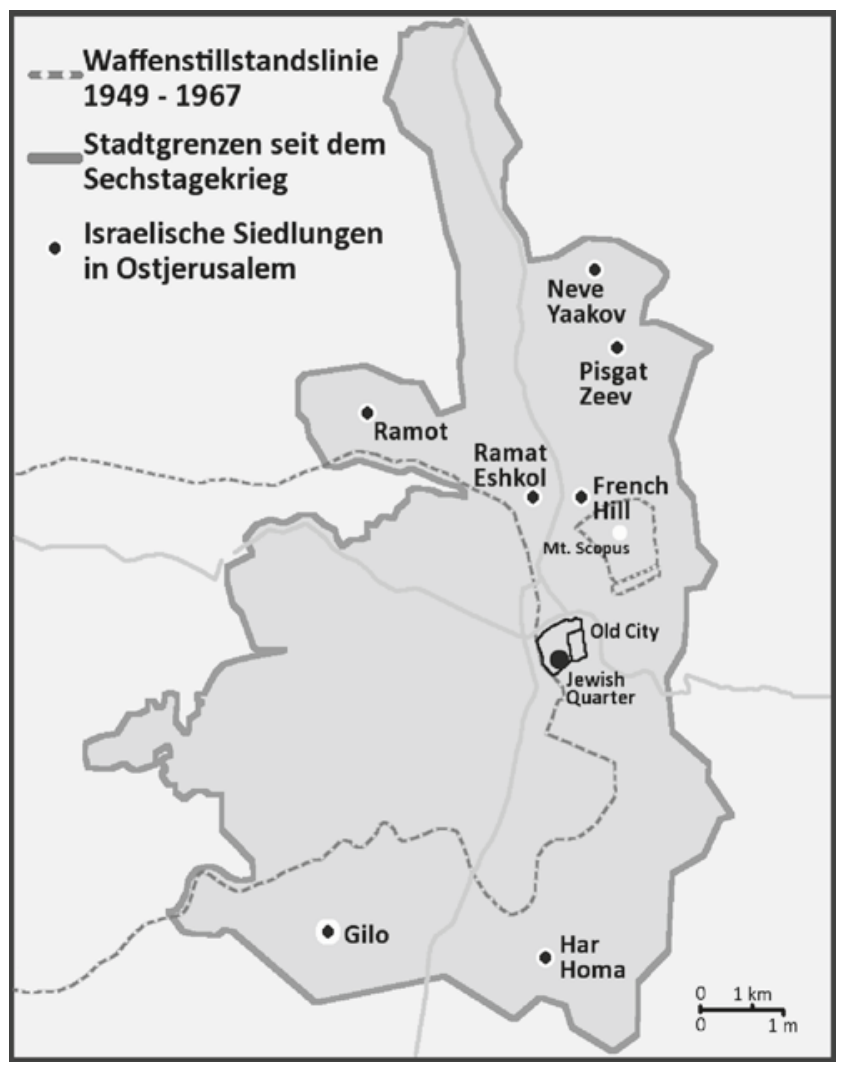

Abb. 2 Jerusalem: Stadtgrenzen und Siedlungen. (Quelle: Israel Ministry of Foreign Affairs (2011), Bearbeitung: Peter Van Gielle Ruppe)

der Klärung des Status Ostjerusalems umfasst neben politischen Machtfragen ebenfalls Aspekte der Bedeutung von religiösen Heiligtümern und ihrer Verortung. Des Weiteren lebten im Jahr 2008 als eine Folge der Jerusalemer Stadtplanung rund 193.000 Israelis in Ostjerusalem (CIA 2011). Damit hat sich die demographische Situation in der ehemals rein palästinensischen Hälfte der Stadt durch die politische Stadtplanung und -entwicklung gravierend verändert. Dennoch ist in der Gesamtstadt Jerusalem aus israelischer Perspektive eine bedrohliche demographische Entwicklung zu beobachten.

\subsection{Bevölkerungspolitik durch Stadtplanung: \\ Baugenehmigungen und Flächennutzungsplanung}

Die Palästinenser Ostjerusalems weisen nicht die im restlichen Staat Israel für Araber geltenden vollen Bürgerrechte auf, verfügen jedoch über ein Wahlrecht auf lokaler Ebene für den Stadtrat. Dieses wird aufgrund einer politischen Kollektiventscheidung bislang kaum in Anspruch genommen, wie folgender Interviewausschnitt erläutert.

You know you are acknowledging the occupation of Eastern Jerusalem. How can we talk that Eastern Jerusalem is occupied and always say that occupation should end and participate the same time. It is a political issue. How can you go and vote for the municipality, which is a municipality of the occupation? So, you can not. (Int. Z. O.)

Die Bevölkerungszahl der wahlberechtigten Palästinenser wäre im Fall einer Inanspruchnahme ihres Wahlrechts bereits ausreichend, um einen signifikanten Einfluss auf die Jerusalemer Stadtpolitik zu nehmen. Weiterhin sind in Ostjerusalem sowie im gesamten Westjordanland ${ }^{2}$ derzeit 37,3 \% der Palästinenser jünger als 14 Jahre alt, so dass in den kommenden Jahren zahlreiche potenzielle Wähler hinzukommen würden (CIA 2011). Somit entsteht für Israel in Anbetracht der demographischen Entwicklung zunehmend das Risiko des Verlustes an demokratisch legitimierter politischer Macht in der Stadt. Problematisch aus israelischer Sicht ist zusätzlich, dass die Zahl der jüdischen Bevölkerung in Jerusalem rückläufig ist (Yiftachel/Yacobi 2002: 138). Für säkulare Juden erscheint Jerusalem aufgrund einer dominierenden orthodoxen Religionsauslegung, der permanent angespannten politischen Situation sowie der wirtschaftlichen Rückständigkeit anderen israelischen Städten gegenüber als wenig attraktiv.

Das Bevölkerungswachstum der Palästinenser Ostjerusalems ist für Israel nicht nur ein lokales Problem in der Hauptstadt, sondern ebenso auf nationaler Ebene von Bedeutung. Die eingeschränkten Rechte der palästinensischen Bevölkerung in Ostjerusalem sind in Anbetracht des israelischen Selbstbildes eines egalitären Rechtsstaates auch in der innenpolitischen Situation riskant, denn sie sind lediglich vor dem Hintergrund einer ausstehenden Klärung der Frage nach Implementierung eines Palästinenserstaates und seiner Ausgestaltung aufrechtzuerhalten. Sollte am Ende dieses Prozesses Ostjerusalem unter israelischer Kontrolle verbleiben, würde dies eine Verleihung der vollen Bürgerrechte für die dort lebenden Palästinenser erfordern und sie somit zur Teilnahme am nationalen politischen Geschehen berechtigen. Weiterhin würde es sie bzgl. ihres Status mit den rund 1,2 Mio. Palästinensern mit israelischer Staatsbürgerschaft gleichstellen, die bereits auf eine lange Tradition der Partizipation am nationalen politischen System zurückblicken können. Bei einer Gesamtbevölkerung von etwa 7,5 Mio. wären die 240.000 weiteren Palästinenser mit israelischer Staatsbürgerschaft und deren höheren Geburtenraten langfristig also auch in der nationalen israelischen Politik von Gewicht (Int. M. M.).

Die Demographie Ostjerusalems weist somit lokale und nationale politische Konsequenzen auf und ist darüber hinaus entscheidend für die Klärung des Status des östlichen Stadtgebiets selbst. Die Ansprüche der Palästinensischen

${ }^{2}$ Separate Daten über die Altersverteilung der palästinensischen Bevölkerung Ostjerusalems lagen nicht vor. 
Abb. 3 Israelische Siedlung Har Homa im südlichen Ostjerusalem, 1997 errichtet inmitten palästinensischer Nachbarschaften. (Fotografie: Peter Van Gielle Ruppe)

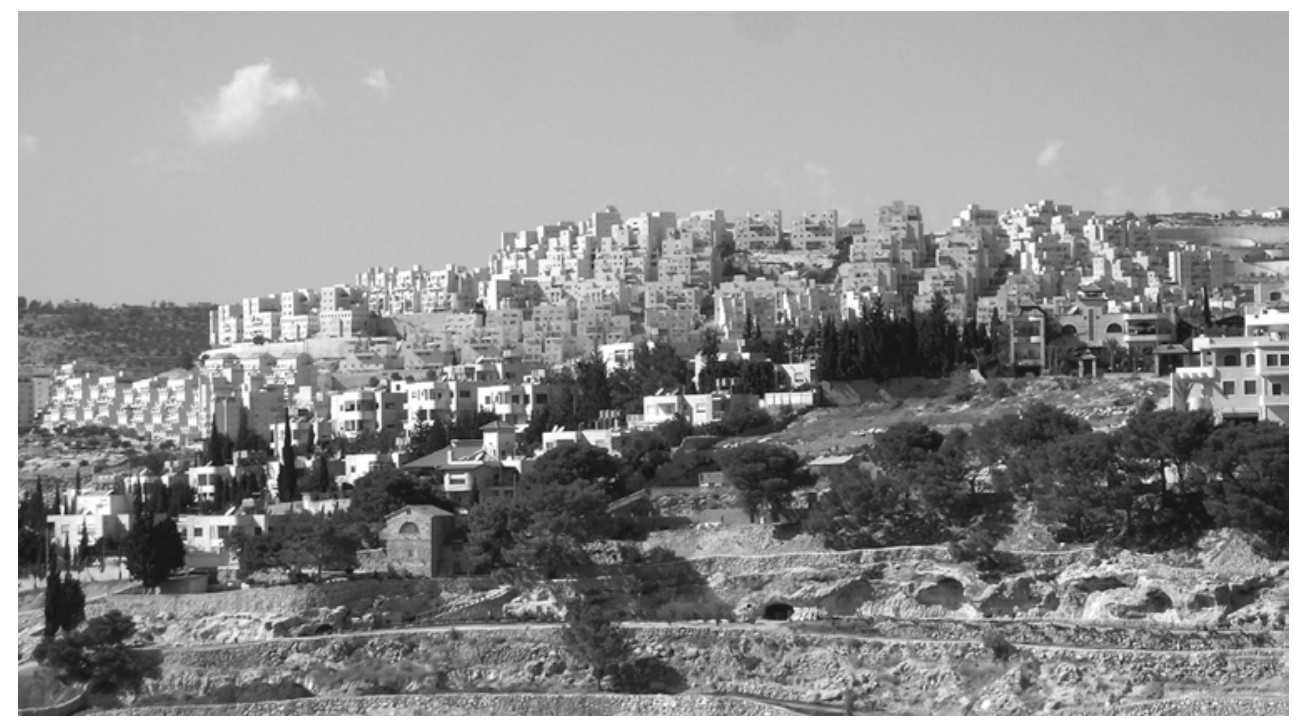

Autonomiebehörden bzgl. Ostjerusalems als Hauptstadt eines Palästinenserstaates sind formal lediglich aufgrund der Anwesenheit einer palästinensischen Bevölkerung aufrechtzuerhalten. Entsprechend wäre eine deutliche jüdische Dominanz im Kontext des Bevölkerungsverhältnisses im Hinblick auf eine internationale Anerkennung der Annexion durch Israel von großem Vorteil. Hieraus ergeben sich für die Stadtadministration zwei anzustrebende Effekte auf die Demographie Jerusalems - eine Zuwanderung jüdischer sowie eine Abwanderung palästinensischer Bevölkerung in Ostjerusalem. An eben dieser Stelle gewinnt eine politisierte oder politische Stadtplanung ihren strategischen Wert für die nationalen Interessen Israels. Yiftachel und Yacobi (2002: 138) spezifizieren dies:

Two central Israeli strategies have been a massive construction of an outer-urban ring of Jewish settlements (, satelite neighborhoods'), which now host over half the Jewish population of the city, and a parallel containment of all Palestinian development, implemented through housing demolition and the prevention of immigration or population growth in the city.

Der Bau der jüdischen Siedlungen in Ostjerusalem seit den frühen 70er Jahren folgt der Idee einer forcierten Zuwanderung durch das Bereitstellen von attraktivem Wohnraum. Des Weiteren wurde die zuvor beschriebene demographische Situation der Stadt in der israelischen Öffentlichkeit als allgemeines Anliegen deklariert, so dass die Beteiligung an einer jüdischen Besiedlung Ostjerusalems in Teilen der Gesellschaft als patriotisch und selbstlos erachtet wird (Yiftachel/Yacobi 2002: 138). Der Bau der Siedlungen wird durch eine Reorganisation der Ostjerusalemer Flächennutzungspläne ermöglicht, die bereits kurze Zeit nach der Besetzung initiiert wurde und mit einer umfangreichen Enteignung ansässiger Palästinenser verbunden war. Bei der
Neuplanung Ostjerusalems wurden zunächst mit etwa 23,3 Quadratkilometern Land etwa $35 \%$ der Gesamtfläche für öffentliche Zwecke konfisziert (Braverman 2007: 339). Auf diesen Flächen entstanden seitdem insgesamt 15 israelische Siedlungen (vgl. Abb. 3) mit rund 60.000 Wohneinheiten und 193.000 ausschließlich jüdischen Bewohnern im Jahr 2008 (Tufakji 2009: 46). Entgegen des rechtlichen Hintergrundes der Enteignungen zum öffentlichen Nutzen sind die Siedlungen und die Flächen, auf denen sie sich befinden, im alltäglichen Leben der Palästinenser ohne Bedeutung. Weitere $40 \%$ der Fläche Ostjerusalems wurde von der Jerusalemer Administration als strategische Reserve, Grünflächen oder nicht für Bebauung freigegebene öffentliche Flächen bestimmt. Nach Abzug der durch die Neuplanung nicht mehr verfügbaren sowie bereits bebauten Flächen verbleiben lediglich 11,2\% Ostjerusalems für eine weitere private Nutzung (Braverman 2007: 340).

Die Schaffung von neuem Wohnraum gestaltet sich für die palästinensische Bevölkerung Ostjerusalems als schwierig. Neben den durch eine hohe Nachfrage hervorgerufenen beträchtlichen Grundstückspreisen für das wenige zum Bau freigegebene Land und einer allgemein problematischen wirtschaftlichen Situation bestehen behördliche Hindernisse. Jeder Neubau sowie Ausbau bereits bestehender Gebäude erfordert eine Genehmigung durch israelische Amtsstellen. Die Kosten der Genehmigungen können dabei mitunter an die der Bauvorhaben heranreichen (Int. T. B.). Darüber hinaus kann es nach Beginn des Legalisierungsprozesses eine unbestimmte Zeit dauern, bis eine Genehmigung zum Bau erteilt wird. In vielen Fällen wird sie letztlich ohne die Rückerstattung der vorausgegangenen Kosten verweigert. Des Weiteren ist der Legalisierungsprozess mit einer ausführlichen juristischen Kontrolle der Antragsteller verbunden, was auf Palästinenser oftmals eine abschreckende Wirkung ausübt (Int. O. N.). 
Tab. 1 Gebäudeabrisse in Ostjerusalem zwischen 1994 und 2006. $\mathrm{JM}=$ Jerusalemer Stadtverwaltung, $\mathrm{MOI}=$ Israelisches Innenministerium. (Quelle: Margalit (2006: 7))

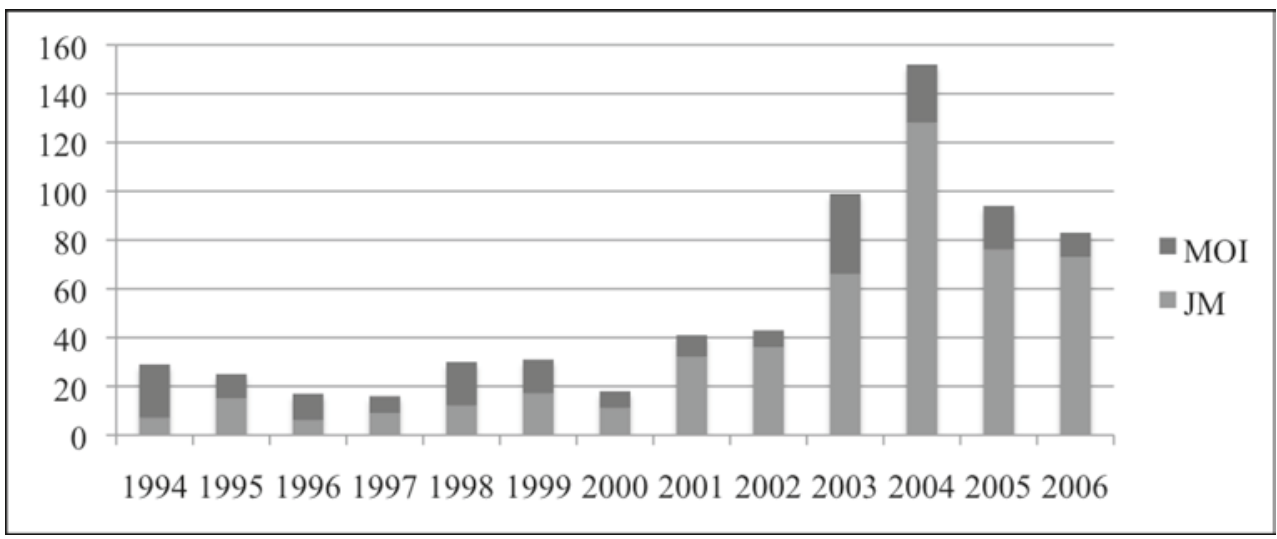

Zwischen 1967 und 1999 wurden lediglich 3.000 Baugenehmigungen an Palästinenser in Jerusalem vergeben. Dies entspricht ungefähr einer Wohnfläche für 25.000 Personen. In derselben Zeit wuchs die palästinensische Population in der Stadt um 112.000 Personen an (Braverman 2007: 339). Diese Entwicklung stellt die palästinensische Gemeinde Ostjerusalem vor ernsthafte humanitäre Probleme. Die durchschnittliche Belegung pro Raum betrug im Jahre 1998 in Ostjerusalem zwei Personen. Rund 23,1 \% der Räume waren mit drei Personen belegt (Arab Studies Society 2004: 21).

Angesichts des Bevölkerungsdrucks und der rechtlichen Rahmenbedingungen, die eine stetig sinkende Lebensqualität der Palästinenser in Ostjerusalem bedingen, gewinnt für diese die Vorstellung der Abwanderung in das Westjordanland an Attraktivität. Dies ist zwar mit einer dauerhaften Aufgabe des Status als Bewohner Jerusalems und all seiner Vorteile verbunden, ermöglicht jedoch in zahlreichen Belangen ein restriktionsfreieres Leben (Int. T. B.). Die Baugenehmigungen als stadtplanerisches Instrument wirken folglich als ein Selektionsmechanismus und üben einen großen Einfluss auf das demographische Verhältnis in der Stadt aus.

Eine weitere Wahlmöglichkeit des Umgangs mit dem Wohnraummangel innerhalb der palästinensischen Gemeinde stellt die Errichtung von Gebäuden ohne die entsprechenden Genehmigungen dar. Dieses illegale Bauen umfasst nicht nur ungenehmigten Neubau, sondern auch den Ausbau bestehender Gebäude sowie die Konstruktion auf nicht dafür vorgesehenen Flächen. In sämtlichen solcher Fälle wird das Gebäude (bzw. der Anbau) von der israelischen Administration als illegal betrachtet, während die verantwortlichen Bauherren eine Straftat begehen. Die Häuserabrisse folgen als politisches Mittel der Stadtplanung konsequent der Linie des restriktiven Umgangs mit Baugenehmigungen. Sie bewirken die Aufrechterhaltung des Bevölkerungsdrucks auf die bestehenden, von Palästinensern genutzten Wohneinheiten in Ostjerusalem und tragen ebenfalls zu dem Effekt einer induzierten Abwanderung in das Westjordanland bei. Darüber hinaus propagie- ren die Abrisse auf symbolischer Ebene die Autorität und territoriale Dominanz der israelischen Besatzungsmacht (Braverman 2007: 346). Laut Angaben der Jerusalemer Stadtverwaltung und des israelischen Innenministeriums liegen derzeit für rund 20.000 bestehende Gebäude, etwa $40 \%$ des palästinensischen Bestandes in Ostjerusalem, keinerlei Baugenehmigungen vor (Braverman 2007: 353).

Tabelle 1 zeigt die Entwicklung der Gebäudeabrisse in Ostjerusalem zwischen 1994 und 2006. Es wird unterschieden, ob die Abrisse der Verantwortung der Jerusalemer Stadtverwaltung (JM) oder dem Israelischen Innenministerium (MOI) unterlagen. Erstere ist verantwortlich für den Abriss ungenehmigter Gebäude auf für Bebauung vorgesehenem Land, wogegen letzteres für den Abriss illegaler Gebäude auf Grünflächen und offenen Flächen verantwortlich ist.

Die Statistik enthält keine Abrisse, die von den Bewohnern selbst vorgenommen wurden. Der Selbstabriss ist oftmals eine Kompromisslösung, bei der den Behörden der Aufwand des Rückbaus erspart bleibt, während die Hausbewohner mit milderen Strafen rechnen können. Anzumerken ist weiterhin, dass die Anzahl der abgerissenen Gebäude ab 2004 abnimmt, die Summe der zerstörten Wohnfläche dagegen ansteigt. Ab 2005 konzentrieren sich die Abrisstätigkeiten verstärkt auf größere Gebäude. Dies hatte in diesen beiden Jahren einen Anstieg der zerstörten Wohnfläche von 9.000 auf 12.000 Quadratmeter zur Folge (Margalit 2006: 7).

Die stadtplanerischen Maßnahmen zur Regulierung des palästinensischen Wohnraums in Ostjerusalem fungieren letztlich als politische Praktiken, um eine Abwanderung der palästinensischen Bevölkerung in das Westjordanland $\mathrm{zu}$ forcieren (Klein 2005: 56). Der auf diesem Wege ausgeübte Druck auf die palästinensische Bevölkerung Ostjerusalems konnte jedoch bislang nicht deren natürliches Bevölkerungswachstum kompensieren, das in Verbindung mit einer stagnierenden Entwicklung jüdischer Bevölkerung zu einer kontinuierlichen Verschiebung des Bevölkerungsverhältnisses zu Gunsten der Palästinenser geführt hat (Int. O. N.). 


\begin{abstract}
Abb. 4 Blick von der City of David auf die palästinensischen Wohnbauten im Tal Silwan. Die Tafel als Teil des archäologischen Parks, der ,City of David", verweist auf die alten Königsgräber, die sich unterhalb der palästinensischen Wohngebäude befinden - die symbolische Reklamation des Gebiets. (Fotografie: Ilse Helbrecht)
\end{abstract}

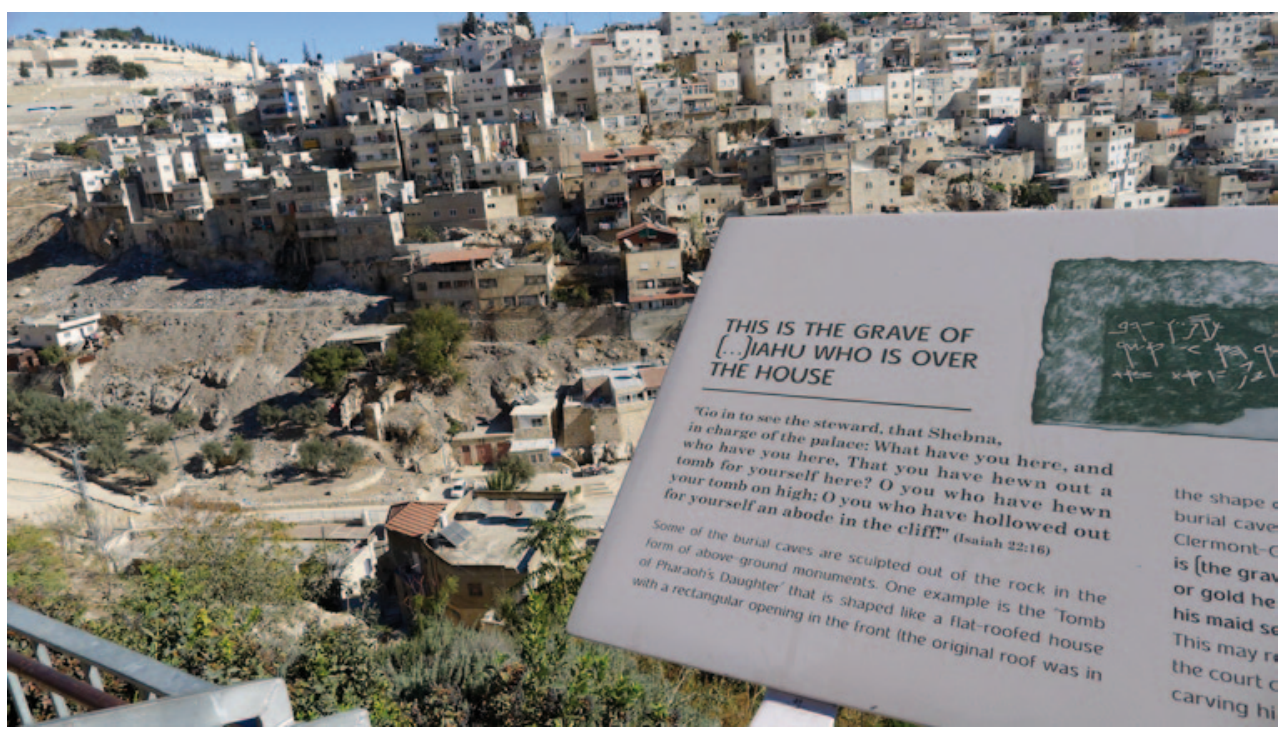

Trotz der daraus resultierenden erschwerten Lebensbedingungen verbleiben weite Teile Ostjerusalems unbestritten palästinensische Wohngebiete. Entsprechend dem israelischen Ziel, durch eine Beeinflussung der Demographie Fakten zu schaffen, um so die Zukunft zu gestalten, bleibt die hohe Präsenz der Palästinenser in Ostjerusalem als geopolitisch bedeutsame Tatsache bis auf weiteres bestehen.

\subsection{Politische Stadtplanung durch Städtebau, Ästhetik und Infrastruktur}

Jerusalem ist aufgrund diverser religiöser und historischer Aspekte ein zentraler Teil der kollektiven israelischen Identität, der wesentliche Beweggrund für die Errichtung des Staates Israel im Heiligen Land und das unbestrittene nationale Symbol (Zuckermann 2009: 30). Khamaisi (2002: 279) beschreibt die nationalen und stadtplanerischen Konsequenzen dieses Sachverhaltes folgendermaßen:

... although geographically peripheral it is Israel's capital. Israeli planning and development policies have been used to strengthen Jerusalem as the Israeli capital and enhance Jewish character.

Hieraus ergibt sich, dass die Verwaltung Jerusalems bei der Stadtplanung die Anwesenheit jeglicher anderer Bevölkerung als der jüdischen weitestgehend ignoriert (Klein 2005: 54). Das Hinwegsetzen über die kulturellen und gestalterischen Traditionen der palästinensischen Mehrheit in Ostjerusalem dient dem politischen Ziel der Etablierung eines Stadtbildes, das eine kulturelle Zugehörigkeit des Stadtgebietes zu Israel und seiner jüdischen Kultur reklamiert. Die „Israelisierung“ des Stadtbildes entspricht auf ästhetischer, städtebaulicher Ebene den zuvor genannten funktionalen und quantitativen Bestrebungen Israels zur Modifizierung der Bevölkerungszusammensetzung Ostjerusalems. Der in Ostjerusalem ent- stehende Raum suggeriert eine beständige Zugehörigkeit zum Staat Israel und dessen jüdischer Bevölkerung (vgl. Abb. 4). Symbolische Raumaneignung und Identitätspolitiken mit den Mitteln des Städtebaus werden so von der nationalen und lokalen Politik zur Sicherung der Stadt Jerusalem als Hauptstadt im Staat Israel genutzt.

Das Verfolgen dieses stadtplanerischen Paradigmas beschränkt sich dabei nicht auf die Siedlungen, sondern tangiert auch die Infrastruktur. So verlaufen die Routen der neuen Jerusalemer Straßenbahn lediglich durch den Westteil der Stadt und einige der größeren jüdischen Siedlungen im Osten. Ein Anschluss an palästinensische Wohngebiete fand in der Planung keinerlei Beachtung, sondern erfolgte meist einzig aufgrund der Lage zwischen israelischen Siedlungen, wie im Fall des nördlich der Altstadt gelegenen Stadtteils Shuafat (Rekacewicz/Vidal 2007). Neben einer infrastrukturellen Benachteiligung der Palästinenser handelt es sich darüber hinaus um eine symbolische Okkupation öffentlichen Raums. Genau wie die israelischen Siedlungen im Ostteil Jerusalems weist die Straßenbahn in ihren äußeren Merkmalen keinerlei Gemeinsamkeiten mit palästinensischen gestalterischen Traditionen auf. Die Fahrpreise orientieren sich an der ökonomischen Realität jüdischer Jerusalemer, so dass die palästinensische Bevölkerung weiterhin auf das eigenständige Ostjerusalemer Busnetzwerk zurückgreifen muss (Rekacewicz/Vidal 2007). Weiterhin ist zu erwarten, dass aufgrund der Erfahrungen mit Terroranschlägen im öffentlichen Nahverkehr Jerusalems die Straßenbahn eine erhöhte Präsenz von israelischen Sicherheitskräften in Ostjerusalem mit sich bringen wird. Die Gegenwart der israelischen Armee führt dabei den Palästinensern symbolisch eine Machthierarchie zwischen den beiden Gruppen vor Augen (Rekacewicz/Vidal 2007).

Ähnlich den zuvor diskutierten stadtplanerischen Maßnahmen zur Einflussnahme auf das Bewohnerverhält- 
Tab. 2 Städtische Einrichtungen in Jerusalem. (Quelle: Yaron (2008: 168))

\begin{tabular}{lcc}
\hline Städtische Einrichtungen in & $\begin{array}{c}\text { Westjerusalem und } \\
\text { Siedlungen im Ostteil }\end{array}$ & $\begin{array}{c}\text { Palästinensische } \\
\text { Nachbarschaften }\end{array}$ \\
\hline Öffentliche Schwimmbäder & 36 & 0 \\
Parks & 1.079 & 45 \\
Bibliotheken & 26 & 2 \\
Sportplätze & 531 & 33 \\
Bewohner pro Kilometer & 690 & 2.917 \\
Bürgersteig & & \\
Bewohner pro & 185 & 4.489 \\
Müllabfuhrwagen & & \\
Bewohner pro öffentlichem & 39 & 5.641 \\
Mülleimer & & \\
\hline
\end{tabular}

nis zeigt sich eine Parallele in der Präsenz der jeweiligen Bevölkerungsgruppen im Stadtbild. Auf der einen Seite wird die Ausbreitung eines israelisch geprägten Jerusalems mit Hilfe der Stadtplanung sukzessiv vorangetrieben, auf der anderen Seite beschränken infrastrukturelle Versäumnisse das palästinensische Leben in der Stadt. Im Jahr 2010 verwendete die Jerusalemer Stadtverwaltung $9 \%$ ihres Budgets auf die Wohngebiete der palästinensischen Bevölkerung, die hingegen $35 \%$ der Gesamtbevölkerung Jerusalems beherbergen (Int M. M.). Die langjährige Praxis einer finanziellen Benachteiligung des ärmeren Ostjerusalems hat eine Schieflage in der öffentlich-städtischen Versorgung zur Folge. Rund $90 \%$ der Abwasserrohre, Bürgersteige und Straßen der Stadt befinden sich in jüdisch dominierten Stadtteilen. 50 \% der von Palästinensern bewohnten Häuser sind ohne Zugang zum Abwassernetz. Lediglich 1,2 \% des Kulturetats sind für die palästinensische Bevölkerung vorgesehen (Yaron 2008: 168). Die asymmetrische Verteilung veranschaulicht (Tab. 2).

Die zahlreichen in Ostjerusalem bestehenden infrastrukturellen Mängel steigern den Abwanderungsdruck auf die Palästinenser und erhöhen den Kontrast zum sich gleichzeitig ausbreitenden charakteristisch-israelischen Stadtbild. Die palästinensischen Nachbarschaften wirken zunehmend wie ein vernachlässigter Fremdkörper inmitten eines systematisch angelegten, von jüdischer Symbolik und Kultur geprägten Jerusalems.

Traditionell stellt Jerusalem den ökonomischen, religiösen und kulturellen Mittelpunkt für Palästinenser dar (Int. T. B.). Per städtische Doktrin werden jedoch generell jegliche politischen und damit faktisch ein Großteil an kultureller und religiöser Aktivität unterbunden (Israel Ministry of Foreign Affairs 2001) (Int. Z. O.). Seit dem Bau der Sperranlagen besteht eine weitere leistungsfähige Möglichkeit zur Kontrolle der Einreisen nach Jerusalem aus dem Westjordanland. Diese ist Palästinensern lediglich als Bürger Jerusalems gestattet und für die Bevölkerung des Westjordanlands nur in
Ausnahmefällen möglich. Im Allgemeinen geht die israelische Administration restriktiv mit diesen Sondergenehmigungen um. Diese umfassen unter anderem Aufenthalte aufgrund medizinischer Notwendigkeiten oder zu Wallfahrtszwecken. Genehmigungen für Wallfahrten werden nur unter weiteren Einschränkungen in Hinblick auf Alter und Geschlecht erteilt, wie z. B. die Erlaubnis des Besuchs der Al-Aqsa Moschee und des Felsendoms an muslimischen Feiertagen, zwei bedeutenden Symbolen islamischer Kultur im historischen Stadtkern Jerusalems. Diese Ausnahmefälle werden üblicherweise von massiven israelischen Sicherheitsvorkehrungen und zahlreichen Mobilitätsrestriktionen für sämtliche Palästinenser in der Altstadt Jerusalems begleitet. Die symbolische Bedeutung Jerusalems als Ort palästinensischer Kultur wird damit über zahlreiche Restriktionen eingeschränkt. Demonstrativer Widerstand von Seiten der ausgeschlossenen Palästinenser gegen das Unzugänglichmachen der religiösen Wallfahrtsorte findet in Form einer Verlagerung der Freitagsgebete in den öffentlichen Raum vor den Mauern der Altstadt oder vom Westjordanland kommend sogar vor den Kontrollpunkten der Sperranlagen statt (Int. T. B.). Weiterhin werden in der Altstadt abgelegene Teile des muslimischen Viertels durch ihre Bewohner (ohne eine rechtliche Grundlage hierzu aber durch die Autoritäten oftmals geduldet) für Andersgläubige unzugänglich gemacht (Int. Z. O.). Dieses Verhalten kann als entgegenwirkende Beanspruchung des Raums angesehen werden und als Indiz aktiven Protests.

Eine direkte Okkupation von Raum findet sich in Form der jüdischen Außenposten in palästinensischen Stadtteilen wieder. Diese Vorposten werden in Folge erzwungener Besitzwechsel von bestehender Bausubstanz errichtet und enthalten damit vergleichsweise mehr Konfliktpotenzial als die neu entstandenen Siedlungen auf enteignetem, aber zuvor unbewohntem Land. Die Wohnsituation der Siedler in verstreuten Enklaven inmitten der als feindlich erachteten palästinensischen Nachbarschaften ist nur in Verbindung mit hohen Kosten für Sicherheitszwecke aufrechtzuerhalten. In der Folge kommt es immer wieder zu Zwischenfällen wie Ausschreitungen zwischen Palästinensern und den israelischen Sicherheitskräften, Massenverhaftungen, Razzien, Steinwürfen auf jüdische Häuser sowie Fahrzeuge. Die Symbolwirkung der Außenposten beschränkt sich nicht auf die bloße Tatsache der national-religiösen Zugehörigkeit der Bewohner, sondern wird durch ein entsprechendes Erscheinungsbild symbolisch verstärkt und kommuniziert (vgl. Abb. 5).

Die Besiedlung vormals palästinensischer Stadtteile durch jüdische Siedler ${ }^{3}$ erfolgt innerhalb von so genannten

\footnotetext{
${ }^{3}$ Die Semantik des Siedlers beinhaltet bereits eine starke Hierarchisierung zwischen den kulturell überlegenen Siedlern und den Anderen. Paul Siu bringt dies zum Ausdruck, wenn er den Siedler wie folgt charakterisiert. Der Siedler ,moves to a country where there is, more or less, a frontier and where the natives have had their own culture but not anything that we may call civilization" (Siu 1952: 44).
} 


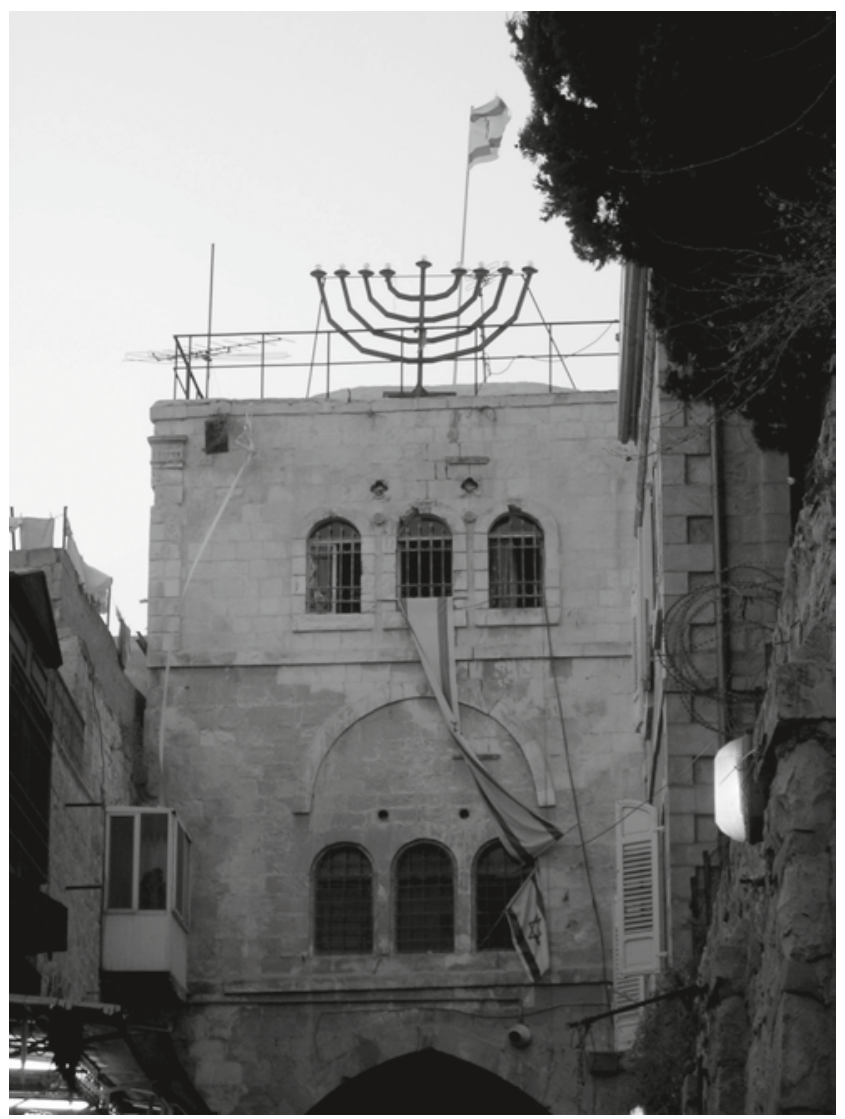

Abb. 5 Siedler Vorposten in der Al-Wad Street, einer der zentralen Verkehrsachsen im muslimischen Viertel der Jerusalemer Altstadt. (Fotografie: Peter Van Gielle Ruppe)

Siedlervereinigungen. Diese zeigen sich für die zumeist gegen den Widerstand der Palästinenser durchgesetzten Hausübernahmen verantwortlich. Das hierzu notwendige restriktive Vorgehen wird oftmals mithilfe einer rechtlich umstrittenen Zusammenarbeit mit israelischen Behörden ermöglicht (Int. M. M.). Diese Vorgänge gehen nicht auf die Initiative von staatlichen Stellen zurück, werden von diesen aber unterstützt, da sie letztlich Regierungsinteressen entsprechen. Der israelische Staat vermeidet eine direkte Verantwortung für das mitunter konfliktreiche Vorgehen bei den Hausübernahmen, das auch national häufig Gegenstand von Kontroversen ist. Die Siedlervereinigungen sind letztlich eine Möglichkeit des israelischen Staates zur institutionellen Auslagerung territorialer Okkupationen. Siedlervereinigungen operieren in einer quasi symbiotischen Beziehung mit der israelischen Regierung in Fragen der symbolischen Kontrolle des Raums (Int. S. E.) (zum Aspekt der räumlichen Kontrolle vgl. statt vieler Stichweh 2008). Ein Interviewpartner (Int. I. S.) führt hierzu aus:

There is a formal government in Israel. They have their policies, but they are a state and they have agreements, international laws. So they give it to semi offi- cial organizations like ELAD to do the work. And they get anything that they want from the ministers. They will agree with any planning that they do. They give them electricity if they need etc.

Die im Zitat benannte Siedlervereinigung ELAD ${ }^{4}$ ist südlich der Jerusalemer Altstadt im palästinensischen Stadtteil Silwan tätig und als Dachorganisation neben zahlreichen Außenposten ebenfalls für den dort befindlichen archäologischen Park „City of David“ verantwortlich. Silwan befindet sich an der Stelle des bronzezeitlichen Jerusalems und war möglicherweise der Regierungsstandort König Davids aus der Zeit des 10. und 11. Jahrhunderts v. Chr. sowie nachgewiesenermaßen das Zentrum des späteren jüdischen Königreiches aus der Zeit zwischen dem 8. und 6. Jahrhundert v. Chr. (Ir Amim 2009: 7). Die „City of David“ firmiert als Symbol der Zugehörigkeit des umgebenden Landes zum jüdischen Volk. Trotz der Kritik von Historikern und Archäologen an der Verbreitung unbelegter Interpretationen im Hinblick auf zahlreiche Exponate (Ir Amim 2009: 27) sind Besuche zu Bildungszwecken fester Bestandteil von Weiterbildungsprogrammen innerhalb staatlicher Organisationen Israels. So verwendet die Siedlervereinigung ELAD bspw. einen beträchtlichen Teil ihres Etats für kostenlose Führungen mit Wehrdienstleistenden der israelischen Armee in Koordination mit deren „Education and Youth Corps" (Ir Amim 2009: 29). Diese mithilfe der „City of David“ implementierte symbolische Verbreitung des historischen Vorrechts einer jüdischen Nation auf Israel, die sich auf die hohe Frequentierung seitens nationaler und internationaler Besucher des archäologischen Parks stützt, geht weit über die Stadtgrenzen Jerusalems hinaus und beeinflusst die politischen Verhältnisse in der Region langfristig.

\subsection{Stadtplanung als politische Planung in Jerusalem}

Die empirischen Ergebnisse zeigen deutlich die politisch motivierten Einflüsse der Jerusalemer Stadtplanung auf quantifizierbare Entitäten wie Bevölkerung und Fläche, auf strukturelle Ausstattungsstandards in der Infrastruktur sowie wahrnehmbare Aspekte wie das Stadtbild und die Raumästhetik. Die Instrumente der Jerusalemer Stadtplanung sind demnach sowohl technisch-infrastrukturelle, symbolische als auch hochgradig politische. Alle drei Momente der Maßnahmen produzieren die skizzierten gesellschaftlichen Effekte einer performativen Herstellung der geographischen Imagination Jerusalem. In Abb. 6 sind die Wirkungsweisen und Zielgrößen der behandelten stadtplanerischen Maßnahmen skizzenhaft systematisiert, wobei zwischen demographischen und stadtbildnerischen Zielgrößen sowie

\footnotetext{
${ }^{4}$ ELAD ist ein hebräisches Akronym und bedeutet in der englischen Übersetzung ,To the City of David“.
} 


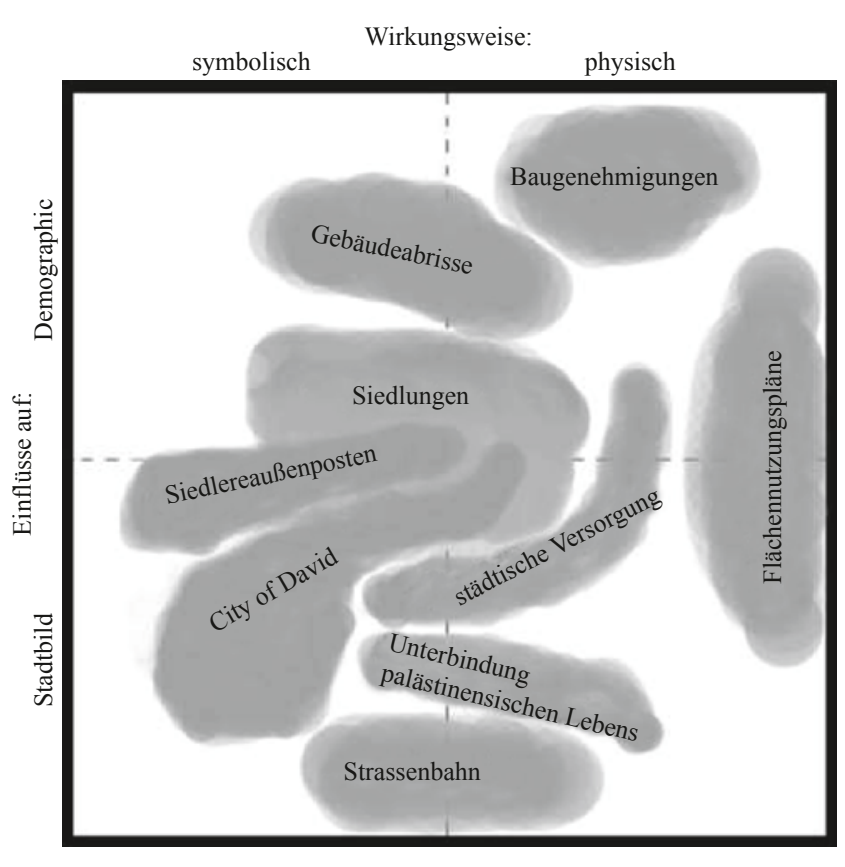

Abb. 6 Politisch einschlägige Zielgrößen und Wirkungsweisen stadtplanerischer Maßnahmen in Jerusalem. (Darstellung: Peter Van Gielle Ruppe)

symbolischer und physischer Wirkungsweise unterschieden wird. Eine physische Wirkungsweise beschreibt direkte materielle Auswirkungen auf die Zielgröße, wohingegen eine symbolische über teilweise demonstrative und teilweise suggestive Einflüsse agiert, die ihre Effekte erst in Folge der Wahrnehmung durch beteiligte Akteure entfalten kann.

Trotz der Unterschiede bzgl. der Wirkungsweise und den zu beeinflussenden Zielgrößen der jeweiligen stadtplanerischen Instrumente zeigen sich deutlich gemeinsame Schnittmengen, die auf ein komplexes Geflecht von Wechselwirkungen hinweisen. Die in der Abbildung dargestellten stadtplanerischen Instrumente mit genuiner politischer Wirkung erheben nicht den Anspruch der Vollständigkeit. Dennoch zeigt sich anhand der dargelegten Beispiele der Demographie- und Infrastrukturbeeinflussung das enorme politische Potenzial von scheinbar technizistischer Planung. Zahlreiche Interviewpartner verwendeten in diesem Zusammenhang den Begriff „facts on the ground“ (u. a. Int. S. A./Int. O. S./Int. Z. O.), der das Vorausgehen einer physischen Realität gegenüber einer nachträglichen Legalisierung beschreibt. Demzufolge ist die verweigerte Anerkennung der israelischen Annexion Ostjerusalems durch die internationale Gemeinschaft kein Hindernis für die Durchführung stadtplanerischer Maßnahmen, da gerade erst deren unbestreitbares Fortschreiten eine nachträgliche Legitimierung und somit Änderung des Status der Stadthälfte bewirken soll.

Die politischen Effekte der Jerusalemer Stadtplanung sind ebenfalls in einer skalaren, also maßstäblichen Perspektive von Interesse. Die angeführten Maßnahmen lassen

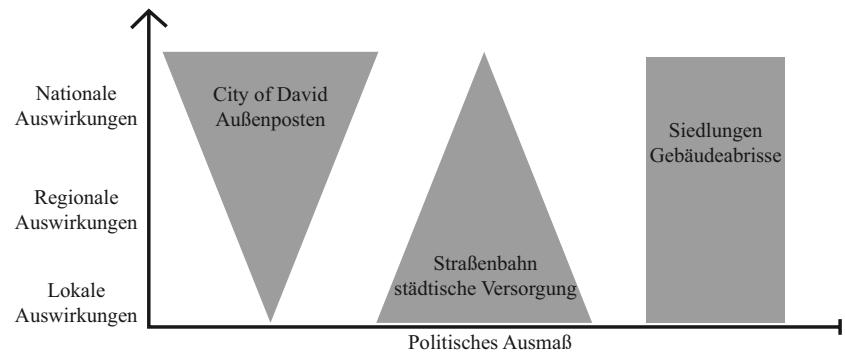

Abb. 7 Politische Effekte stadtplanerischer Maßnahmen in Abhängigkeit von der betrachteten Skala. (Darstellung: Peter Van Gielle Ruppe)

sich in Hinblick auf ihre deutlichste räumliche Ausprägung spezifizieren. Während die jüdischen Außenposten und die „City of David“ evident auf ein nationales Skalenniveau gerichtet sind und auf eine symbolische Wirkung zur Aufrechterhaltung des Staates Israel zielen, wirken die direkten stadtplanerischen Maßnahmen wie die Streckenführung der Straßenbahn in Jerusalem oder die Ver- und Entsorgung der Stadträume besonders auf dem lokalen Skalenniveau. Die politische Absicht zeigt sich hier weniger symbolisch als vielmehr materiell und existenziell auf die jeweiligen Lebensumstände in den Teilräumen der Stadt gerichtet. Die stadtplanerischen Maßnahmen wie Gebäudeabrisse oder die Errichtung von Siedlungen wirken dagegen sowohl symbolisch auf nationalem als auch physisch auf lokalem Skalenniveau. Der Einfluss verschiedener Maßnahmen in Abhängigkeit von dem betrachteten Skalenniveau ist in Abb. 7 dargestellt.

Die stadtplanerischen Maßnahmen in Jerusalem sind demnach in einer Wechselbeziehung von nationalen Wirkungen und lokalen Effekten verortet. Ihre evidente politische Bedeutung liegt in der Manifestation von einer geographischen Imagination Jerusalem als Hauptstadt Israels, die über verschiedene auf lokaler wie nationaler Ebene angesiedelter Instrumente reifiziert wird. Stadtplanung in Jerusalem dient demnach meist auch der Konstituierung und Implementierung einer genuin politischen nationalen israelischen Idee.

\section{Schlussbetrachtung: Stadtplanung als performativer politischer Prozess in Jerusalem}

Jerusalem bietet eine eindrückliche Fallstudie zu der umfassenden Wirkmacht einer politisierten Stadtplanung. Stadtplanung ist nicht nur ein lokales Herrschaftsinstrument, sondern wirkt multiskalar, indem ebenso nationale wie geostrategische Interessen hiermit durchgesetzt werden können. Versucht man am Ende dieses Beitrags theoretisch präzise zu identifizieren, was genau Stadtplanung in Jerusalem (und nicht nur dort) zu so einem mächtigen Herrschaftsinstrument des Staates macht, so ist es notwendig, die bis hierher präsentierten empirischen Ergebnisse im Lichte 
aktueller Theorien zum Verhältnis von Raum, Kultur und Macht zu reflektieren. Hierfür greifen wir resümierend auf neuere Erkenntnisse der Kulturgeographie und Performanztheorie zurück.

Die jüngere Geschichte der Stadtplanung von Jerusalem lässt sich als der Versuch lesen, eine jeweils religiös motivierte Ideologie in der baulichen Gestalt der Stadt zu verankern. Dabei kommt es zu Reifikationen dessen, was Derek Gregory in Anlehnung an C. Wright Mills und David Harvey geographische Imagination nennt (Gregory 1994). Die religiös motivierten Vorstellungen der jeweils herrschenden Gruppe im Hinblick auf die soziale wie symbolische Ordnung des Raums schreiben sich über permanente Wiederholungen in die physische Struktur der heiligen Stadt Jerusalem ein. Die räumlichen Konstruktionen der geographischen Imagination und deren Raumabstraktionen weisen eine immanente performative Kraft der Selbstrealisation auf, die sich wiederum stark auf die Akteure auswirkt, die diese geographischen Imaginationen bewohnen, wie Gregory ausführt. „[T]hese abstractions of space are more than mere words; they have performative force, and they bear down on those that inhabit them" (Gregory 2004: 602). Sie werden in einem iterativen Prozess performativ zu Wirklichkeit, d. h., ihre Wiederholung im publizistischen Diskurs, auf Flächennutzungsplänen oder in Geschichtsbüchern trägt dafür Sorge, dass in der physisch-materiellen Welt diese geistigen Konstrukte, z. B. von Territorien, ethnischen Gemeinschaften oder Hierarchien, in der Beziehung von sozialen Gruppen oder Klassen hergestellt werden. Die Auswirkungen dieser performativen Effekte sind letztlich die, dass das Handeln der Entscheidungsträger auf den Vollzug oder die Herstellung der geographischen Imaginationen ausgerichtet wird.

Die heutige Entwicklung von Jerusalem als einer Stadt, die für drei Weltreligionen bedeutsam ist, lässt sich als ein Produkt dieser performativen Akte der Reifikation geographischer Imaginationen deuten, die Gregory in seinen Arbeiten darlegt (Gregory 1994; Gregory 1995a; Gregory 2004). Zunächst orientierten sich die britischen Mandatsträger nach ihrer Übernahme der Stadt am 9. Dezember 1917 nach Westen. Die östlichen Stadtteile von Jerusalem waren von ihrer naturräumlichen Ausstattung und ideologischen Bedeutung her aus Sicht der Briten zu vernachlässigen. Diese konzentrierten sich auf die Altstadt mit ihren heiligen Stätten und den westlichen Gebieten (Efrat/Noble 1988: 392). Die folgende Teilung Jerusalems in einen israelischen und einen jordanischen Teil erklärt die unterentwickelten west-östlichen Verkehrsachsen. Der Plan von 1959 zur Entwicklung Jerusalems sah mit dem „Mandelbaum Gate“ nur einen einzigen Übergang zwischen den Stadtteilen vor (Efrat/Noble 1988: 397). Mit dem Ausbruch des Sechs-Tage-Krieges am 5. Juni 1967 schließlich entschieden Militärs über die Grenzen der wiedervereinigten Stadt Jerusalem.
Diese Grenzziehung wurde durch zwei Aspekte geleitet: der Einbezug von Höhen in das Stadtgebiet zu einer Optimierung der Verteidigungsfähigkeit der Stadt und eine Maximierung der Stadtfläche bei gleichzeitiger Minimierung der arabischen Bevölkerung (Efrat/Noble 1988: 398 ff.). Die dargelegten empirischen Ergebnisse deuten auf eine explizite Fortführung dieser Prämissen in der heutigen Stadtplanung von Jerusalem.

Die empirischen Resultate der Studie zeigen deutlich, dass es sich bei der Stadtplanung in Jerusalem um einen politischen Prozess handelt, in dem über die Durchsetzung verschiedenster raumprägender Maßnahmen eine geographische Imagination Jerusalem performativ hergestellt wird. Diese imaginären Geographien überführen als ihr wesentlichstes Merkmal Distanzen in Unterschiede (Gregory 2003: 307). Geographische Imaginationen sind in der Lage, einen eigenen Raum als unvertraut und feindlich und einen fremden Raum als vertraut entstehen zu lassen. Wesentlich ist an diesen räumlich-geschichtlichen Vorstellungen ihr performativer Charakter. Geographische Imaginationen sind nicht einfache Zeitreihen von räumlich verorteten Ereignissen oder räumliche Sedimentationen historischer Prozesse, sondern räumliche Performanzen im Sinne von Gillian Rose (Rose 1999). Gregory erkennt in den physischen Einschreibungen dieser imaginären Geographien, wie z. B. Grenzbefestigungsanlagen und deren eigene räumliche Gestalt von Zäunen und Mauern sowie den Zwischenräumen, die sie aufspannen, gleichzeitig repetitive Performanzen der Macht. Wesentlich ist in den Arbeiten zu geographischen Imaginationen und deren performativen Effekten gerade dieses ,,vor Augen führen“ von Machtlosigkeit und Machtfülle, die in den täglichen Grenzperformanzen, wie Kontrollen und Leibesvisitationen, Zurückweisungen und Bedrohungen oder in Planungsprozessen und Verwaltungsentscheidungen ihren Ausdruck finden. Letztlich schreiben all diese Machtdemonstrationen den israelischen Machtanspruch performativ in Palästina ein (Gregory 2004: 604). Raum ist in diesem Sinne nicht in einer kantischen Lesart a priori gegeben und die Ermöglichung der Erscheinung der Dinge (Kant 2005 (1787): 72), sondern ein Produkt von raumkonstituierenden Performanzen und damit ein genuines Ergebnis von Macht (Rose 1999: 249). Diese Performanzen, z. B. das kommunizierte Handeln der Jerusalemer Stadtplanung, die Publikation von bestimmten thematischen Karten oder öffentliche Reden von Entscheidungsträgern, erzeugen letztlich einen Raum, in dem das Neue oder das Andere geschärft in die Welt tritt (Gregory 2003: 308). Die israelische Stadtplanung in Jerusalem ist in diesem Sinne ebenfalls eine politische Performanz, die aus städtischen Räumen abstrakte politische Arenen konstruiert, die sowohl symbolische Ansprüche des Eigenen kommunizieren als auch eine Präsenz dieses Eigenen des israelischen Staates konstituieren. Diese bereits performativ konstituierten Räume des Eigenen 
wirken wiederum als Manifestation geographischer Imaginationen ihrerseits performativ. Sie können bspw. von unbescholtenen nicht-israelischen Bürgern betreten werden, die anschließend nur aufgrund dieser Betretung zu Kombattanten aus Sicht der herrschenden Jerusalemer Politik mutieren (Gregory 2003: 312). Konsequenterweise denkt Gregory Möglichkeiten der Überwindung dieser Performanzen räumlicher Machtausübung in Begriffen von Veränderungen der diese Effekte motivierenden geographischen Imaginationen. Im Rückgriff auf die Arbeiten von Homi Bhabha (1994) zur postkolonialen Kulturtheorie verweist Gregory auf die Möglichkeit der Produktion von Gegen-Repräsentationen und Gegen-Wissen als ein bedeutendes Mittel im Kampf gegen mächtige Ideologien, die auf Performanzen und geographischen Imaginationen beruhen. Der postkolonialen Theoriebildung fällt in diesem Zusammenhang eine Schlüsselstellung zu, um im Sinne einer „Aufklärung“ die notwendigen geographischen (Gegen)Imaginationen und (Gegen)Performativa zu entwickeln und zu kommunizieren (Gregory 1995b).

Die in diesem Beitrag als politische analysierten und auf verschiedenen Skalenebenen ansetzenden Instrumente der Stadtplanung in Jerusalem sind performativ in dem Sinne, dass sie die als Hintergrundfolien politischen Handelns existierenden geographischen Imaginationen der Hauptstadt Israels über kontinuierliche iterative Prozesse erst reifizieren. Ihr Ziel ist die Herstellung von Präsenz israelischer Macht, wobei Präsenz auf ein räumliches Verhältnis zur Welt und deren Gegenständen abzielt und nicht auf die Temporalisierung von Anwesenheit. Ist etwas präsent, ist es für den Menschen greifbar und fassbar. Gleichzeitig kann es unmittelbar auf den menschlichen Körper einwirken (Gumbrecht 2005: 11). Stadtplanerische Maßnahmen können so die Präsenz und damit die körperliche und haptische Erfahrbarkeit von staatlicher Macht in bestimmten Räumen konstituieren. Im Beispiel der Stadtplanung in Jerusalem ist diese Implementierung geleitet durch machtvolle geographische Imaginationen, die, folgt man Tamar Mayer, momentan dominieren: „Jerusalem once again has become the promised city, the spiritual center, and a place of prayer" (Mayer 2008: 240). Die in Haifa geborene Geographin israelisch-jüdischer Herkunft, Tamar Mayer, die heute in den USA lebt und lehrt, kommt zu dem Schluss, dass der jüdische Nationalismus heute noch mehr als zu Beginn des 20. Jahrhunderts zunehmend Jerusalem in das Zentrum eines neuen Zionismus rückt (Mayer 2008: 240). Dementsprechend wird sich die Bedeutung Jerusalems für die friedliche Lösung des Nahost-Konflikts nur mehr steigern - und mit ihr die strategische Rolle der Stadtplanung bei der Realisierung imaginierter Geographien einer Hauptstadt. Die Jerusalemer Stadtplanung prägt ganz entscheidend die materielle und ideelle Struktur der städtischen Räume auch im östlichen Teil der Stadt und passt sie über die im empiri- schen Teil explizierten Maßnahmen und deren performative Effekte an tradierte Vorstellungen des israelischen Regierungssitzes an. Die Herstellung Jerusalems als Hauptstadt Israels ist ein nationales, geopolitisches Projekt, an dessen Realisierung die politische Stadtplanung mächtig mitwirkt.

Open Access Dieser Artikel unterliegt den Bedingungen der Creative Commons Attribution License. Dadurch sind die Nutzung, Verteilung, und Reproduktion erlaubt, sofern der/die Originalautor/en und die Quelle angegeben sind.

\section{Literatur}

Aguilar, A. (1987): Urban planning in the 1980s in Mexico City: Operative process or political facade? In: Habitat International 11, 3, 23-38.

Albers, G.; Papageorgiou-Venetas, A. (1984): Stadtplanung. Entwicklungslinien 1945-1980. Band 1: Entwicklungslinien. Tübingen.

Altrock, U. (1998): Stadtplanung in Israel und Palästina. Der Friedensprozeß als Neubeginn? Berlin. = Arbeitshefte des Instituts für Stadt- und Regionalplanung der TU Berlin, Band 60 .

Altrock, U.; Güntner, S.; Kennel, C. (2004): Zwischen analytischem Werkzeug und Politikberatung: ein kritischer Blick auf aktuelle Leitbegriffe in der Stadtentwicklungspolitik. In: Altrock, U.; Güntner, S.; Huning, S.; Peters, D. (Hrsg.): Perspektiven der Planungstheorie. Berlin, 187-207. = Reihe Planungsrundschau, Band 10.

Arab Studies Society (2004): Breaking the siege of denying natural growth of Palestinian neighborhoods in East Jerusalem. Jerusalem.

Bhabha, H. K. (1994): The Location of Culture. London, New York.

Bollens, S. A. (2000): On Narrow Ground: Urban Policy and Ethnic Conflict in Jerusalem and Belfast. Albany.

Braverman, I. (2007): ,Powers of Illegality': House Demolitions and Resistance in East Jerusalem. In: Law and Social Inquiry 32, 2, 333-372.

Brik, N. (1996): Jerusalem. Einflüsse einer ethnisch-differenzierenden Ideologie auf die Stadtentwicklung. Augsburg. = Beiträge zur Angewandten Sozialgeographie, Band 33.

Butler, J. (2006): Haß spricht. Zur Politik des Performativen. Frankfurt am Main.

Christopher, A. J. (1987): Apartheid Planning in South Africa: The Case of Port Elizabeth. In: The Geographical Journal 153, 2, 195-204.

CIA (Central Intelligence Agency) (2011): The World Factbook: West Bank. Online unter: https://www.cia.gov/library/publications/theworld-factbook/geos/we.html (letzter Zugriff am 27.06.2012).

Dumper, M. (1996): The Politics of Jerusalem since 1967. New York.

Efrat, E.; Noble, A. G. (1988): Planning Jerusalem. In: Geographical Review 78, 4, 387-404.

Emmi, P. C.; Santigosa, M. A. (1989): Urban development, land use planning and political change: The case of Costa Brava, Spain. In: Land Use Policy 6, 2, 103-120.

Fischer-Lichte, E. (2004): Ästhetik des Performativen. Frankfurt am Main.

Fürst, D. (2007): Urban Governance. Einblicke in die Diskussion. In: Städte im Umbruch. Das Online Magazin für Stadtentwicklung, Stadtschrumpfung, Stadtumbau \& Regenerierung 4, 5-10.

Gordon, N. (2008): Israel's Occupation. Berkeley.

Gregory, D. (1994): Geographical Imaginations. Cambridge, Oxford.

Gregory, D. (1995a): Imaginative Geographies. In: Progress in Human Geography 19, 4, 447-485. 
Gregory, D. (1995b): Reflection: A Geographical Unconscious: Spaces for Dialogue and Difference. In: Annals of the Association of American Geographers 85, 1, 175-186.

Gregory, D. (2003): Defiled Cities. In: Singapore Journal of Tropical Geography 24, 3, 307-326.

Gregory, D. (2004): Palestine under Siege. In: Antipode 36, 4, 601-606.

Gumbrecht, H. U. (2005): Diesseits der Hermeneutik. Die Produktion von Präsenz. Frankfurt am Main.

Häußermann, H.; Läpple, D.; Siebel, W. (2008): Stadtpolitik. Frankfurt am Main.

Helbrecht, I. (1994): Stadtmarketing - Konturen einer kommunikativen Stadtentwicklungspolitik. Basel, Boston, Berlin.

Helbrecht, I. (2009): Mehr Leadership! Der Weg in die Urban Governance am Beispiel Creative City Politics. Eine Betrachtung im Lichte von Platons Staatstheorie. In: PND-online 1, 1-14.

Ir Amim (2009): Shady Dealings in Silwan. Jerusalem.

Israel Ministry of Foreign Affairs (1980): Basic Law: Jerusalem, Capital of Israel. Online unter: http://www.mfa.gov.il/MFA/MFAArchive/1980_1989/Basic+Law-+Jerusalem-+Capital+of+Israel. htm (letzter Zugriff am 27.06.2012).

Israel Ministry of Foreign Affairs (1999): The Status of Jerusalem. Online unter: http://www.mfa.gov.il/MFA/MFAArchive/1990 1999/1999/3/The+Status+of+Jerusalem.htm (letzter Zugriff am 27.06 .2012 ).

Israel Ministry of Foreign Affairs (2001): Israeli Measures in the Jerusalem Area in Response to Palestinian Terrorism - Legal Aspects. Online unter: http://www.mfa.gov.il/MFA/MFAArchive/2000_2009/2001/8/Israeli\%20Measures\%20in\%20the $\% 20$ Jerusalem\%20Area\%20in\%20Response (letzter Zugriff am 27.06.2012).

Israel Ministry of Foreign Affairs (2011): Israel's Story in Maps. Online unter: http://www.mfa.gov.il $/ \mathrm{mfa} /$ facts\%20about\%20 israel/israel\%20in\%20maps/ (letzter Zugriff am 27.06.2012).

Kant, I. (2005 (1787)): Kritik der reinen Vernunft. Immanuel Kant. Werke in sechs Bänden. Herausgegeben von Wilhelm Weischedel. Band 2. Darmstadt.

Khamaisi, R. (1997): Israeli use of the British mandate planning legacy as a tool for the control of Palestinians in the West bank. In: Planning Perspectives 12, 3, 321-340.

Khamaisi, R. (2002): Shared space, separate geo-politically: Al-Quds Jerusalem capital for two states. In: Geoforum 33, 6, 278-283.

Klein, M. (1999): Doves over Jerusalem's Sky. Jerusalem.

Klein, M. (2005): Old and new walls in Jerusalem. In: Political Geography $24,1,53-76$.

Margalit, M. (2006): No Place Like Home: House Demolitions in East Jerusalem. Jerusalem.

Mayer, T. (2008): Jerusalem in and out of focus: the city in Zionist ideology. In: Mayer, T.; Mourad, S. A. (Hrsg): Jerusalem. Idea and Reality. London, New York, 224-244.
Pahl-Weber, E. (2011): Politikberatung in der Ressortforschung zur Bau-, Stadt- und Raumforschung. In: Informationen zur Raumentwicklung 7/8, 397-408.

Peters, D. (2004): Zum Stand der Planungstheorie. In: Altrock, U; Güntner, S.; Huning, S.; Peters, D. (Hrsg.): Perspektiven der Planungstheorie. Berlin, 5-18. = Reihe Planungsrundschau, Band 10.

Rekacewicz, P.; Vidal, D. (2007): The Politics of Urban Planning: Jerusalem's Apartheid Tramway: In: Le Monde Diplomatique vom 8. Februar 2007. Online unter: http://mondediplo.com/2007/ 02/08tramway (letzter Zugriff am 26.06.2012).

Reuter, W. (2004): Planung und Macht - Positionen im theoretischen Diskurs und ein pragmatisches Modell von Planung. In: Altrock, U.; Güntner, S.; Huning, S.; Peters, D. (Hrsg.): Perspektiven der Planungstheorie. Berlin, 57-78. = Reihe Planungsrundschau, Band 10.

Rose, G. (1999): Performing Space. In: Massey, D.; Allen, J.; Sarre, P. (Hrsg.): Human Geography Today. Cambridge, Malden, 247-259.

Rubin, N. H. (2011): Geography, colonialism and town planning: Patrick Geddes' plan for mandatory Jerusalem. In: Cultural Geographies 18, 2, 231-248.

Schechner, R. (1998): What Is Performance Studies Anyway? In: Phelan, P.; Lane, J. (Hrsg.): The Ends of Performance. New York, London, 357-362.

Schumacher, E. (2002): Performativität und Performance. In: Wirth, U. (Hrsg.): Performanz. Zwischen Sprachphilosophie und Kulturwissenschaften. Frankfurt am Main, 383-402.

Selle, K. (1994): Was ist bloß mit der Planung los? Erkundungen auf dem Weg zum kooperativen Handeln. Dortmund. = Dortmunder Beiträge zur Raumplanung, Band 69.

Sinz, M. (2011): Raumordnung als Gegenstand von Politikberatung ein Erfahrungsbericht. In: Informationen zur Raumentwicklung 7/8, 471-486.

Siu, P. C. P. (1952): The Sojourner. In: American Journal of Sociology $58,1,34-44$.

Stichweh, R. (2008): Kontrolle und Organisation des Raums durch Funktionssysteme der Weltgesellschaft. In: Döring, J.; Thielmann, T. (Hrsg.): Spatial Turn. Das Raumparadigma in den Kultur- und Sozialwissenschaften. Bielefeld, 149-164.

Streich, B. (2011): Stadtplanung in der Wissensgesellschaft. Wiesbaden.

Tufakji, K. (2009): Land Confiscation and Settlement Construction in Occupied East Jerusalem. In: The Civic Coalition for Defending Palestinians' Rights in Jerusalem (Hrsg.): 42 Year of Occupation - Jerusalem File. Jerusalem, 41-67.

Weizman, E. (2007): Hollow Land - Israel's Architecture of Occupation. London.

Winkler, H. (2004): Diskursökonomie. Versuch über die innere Ökonomie der Medien. Frankfurt am Main.

Yaron, G. (2008): Jerusalem - Ein historisch-politischer Stadtführer. München.

Yiftachel, O.; Yacobi, H. (2002): Planning a bi-national capital: Should Jerusalem remain united? In: Geoforum 33, 1, 137-145.

Zuckermann, M. (2009): Sechzig Jahre Israel - Die Genesis einer politischen Krise des Zionismus. Bonn. 